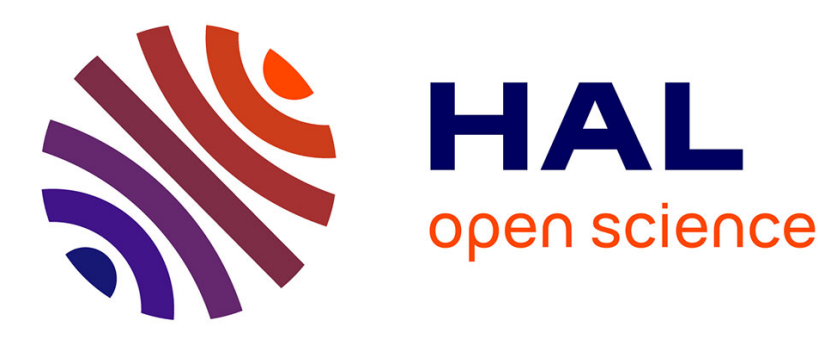

\title{
Glottal behavior in the high soprano range and the transition to the whistle register
}

Maëva Garnier, Nathalie Henrich Bernardoni, Lise Crevier-Buchman, Coralie Vincent, John Smith, Joe Wolfe

\section{- To cite this version:}

Maëva Garnier, Nathalie Henrich Bernardoni, Lise Crevier-Buchman, Coralie Vincent, John Smith, et al.. Glottal behavior in the high soprano range and the transition to the whistle register. Journal of the Acoustical Society of America, 2012, 131 (1 - Part 2), pp.951-962. 10.1121/1.3664008 . hal-00660394

\section{HAL Id: hal-00660394 \\ https://hal.science/hal-00660394}

Submitted on 18 May 2012

HAL is a multi-disciplinary open access archive for the deposit and dissemination of scientific research documents, whether they are published or not. The documents may come from teaching and research institutions in France or abroad, or from public or private research centers.
L'archive ouverte pluridisciplinaire HAL, est destinée au dépôt et à la diffusion de documents scientifiques de niveau recherche, publiés ou non, émanant des établissements d'enseignement et de recherche français ou étrangers, des laboratoires publics ou privés. 


\title{
Glottal behavior in the high soprano range and the transition to the whistle register
}

\author{
Maëva Garnier ${ }^{\text {a) }}$ \\ School of Physics, University of New South Wales, Sydney, New South Wales 2052, Australia \\ Nathalie Henrich \\ Department of Speech and Cognition, Grenoble Images Parole Signal Automatique (UMR 5216 CNRS/Grenoble \\ INP/UJF/U. Stendhal), 12 rue des mathématiques, BP. 46, 38402 Grenoble Cedex, France \\ Lise Crevier-Buchman and Coralie Vincent \\ Laboratoire de Phonétique et Phonologie (UMR 7018 CNRS/Université Paris 3/Sorbonne Paris Cité), \\ 19 rue des Bernardins, 75005 Paris, France \\ John Smith and Joe Wolfe \\ School of Physics, University of New South Wales, Sydney, New South Wales 2052, Australia
}

(Received 25 December 2010; revised 19 October 2011; accepted 24 October 2011)

\begin{abstract}
The high soprano range was investigated by acoustic and electroglottographic measurements of 12 sopranos and high-speed endoscopy of one of these. A single laryngeal transition was observed on glissandi above the primo passaggio. It supports the existence of two distinct laryngeal mechanisms in the high soprano range: $\mathrm{M} 2$ and $\mathrm{M} 3$, underlying head and whistle registers. The laryngeal transition occurred gradually over several tones within the interval D\#5-D6. It occurred over a wider range and was completed at a higher pitch for trained than untrained sopranos. The upper limit of the laryngeal transition during glissandi was accompanied by pitch jumps or instabilities, but, for most singers, it did not coincide with the upper limit of $R 1: f_{0}$ tuning (i.e., tuning the first resonance to the fundamental frequency). However, pitch jumps could also be associated with changes in resonance tuning. Four singers demonstrated an overlap range over which they could sing with a full head or fluty resonant quality. Glottal behaviors underlying these two qualities were similar to the M2 and M3 mechanisms respectively. Pitch jumps and discontinuous glottal and spectral changes characteristic of a M2-M3 laryngeal transition were observed on decrescendi produced within this overlap range.
\end{abstract} (C) 2012 Acoustical Society of America. [DOI: 10.1121/1.3664008]

PACS number(s): 43.75.Rs [DAB]

Pages: 951-962

\section{INTRODUCTION}

Sopranos demonstrate several changes in voice quality during glissandi, often accompanied by pitch jumps or instabilities (Miller, R., 2000). Although the main lower transition (below A4, $440 \mathrm{~Hz}$ ) has been much described by scientific studies, relatively little is known about transitions and registers in the higher range. There is still no consensus on the number of transitions, on the pitch at which they occur, nor on their physical nature.

The first transition in the soprano voice typically occurs around E4-F4 ( $340 \mathrm{~Hz})$ (Miller, D.G., 2000; Miller, R., 2000; Roubeau et al., 2004, 2009; Henrich, 2006). Commonly known as the primo passaggio or the chest-head register transition, this transition corresponds to the M1-M2 change in laryngeal mechanism (Roubeau et al., 2009). Classical sopranos are trained to lower this transition to avoid a voice "break" and changes in quality toward the bottom of their tessitura. Consequently, they often extend the range of the M2 laryngeal mechanism to pitches as low as C4 $(\sim 260 \mathrm{~Hz})$.

\footnotetext{
a) Author to whom correspondence should be addressed. Electronic mail: maeva.garnier@gipsa-lab.grenoble-inp.fr
}

A second transition somewhere in the range C5-G5 $(\sim 500-700 \mathrm{~Hz})$ is mentioned by some authors. Known as the secondo passaggio, this transition divides the C4-C6 range into a middle and an upper register (Sonninen et al., 1999; Miller, D.G., 2000; Miller, R., 2000; Echternach et al., 2010). Some authors suggested that this transition could be related to vocal-tract tuning (Miller, D.G., 2000). Indeed, B4-D5 corresponds approximately to the pitch range where the fundamental frequency $\left(f_{0}\right)$ reaches the frequency range of the first vocal tract resonance for closed and mid vowels and above which sopranos tune the frequency of their first vocal tract resonance $(R 1)$ to the fundamental frequency $\left(f_{0}\right)$ (Sundberg, 1975; Joliveau et al., 2004a; Garnier et al., 2010). In some sopranos, B4-D5 also corresponds to the pitch range from which they start increasing mouth aperture (Sundberg and Skoog, 1997; Echternach et al., 2010; Garnier et al., 2010). Another study showed significant changes in distance and position of laryngeal structures, supporting the idea that the secondo passaggio corresponds to a significant change in laryngeal behavior and pitch control mechanism (Sonninen et al., 1999).

A third transition is commonly reported in the top range of the soprano voice, occurring somewhere in the broad range E5 $(660 \mathrm{~Hz})$ to $\mathrm{G} 6(1570 \mathrm{~Hz})(B e h n k e, 1880$; Van den 
Berg, 1963; Van Deinse, 1981; Shipp et al., 1988; Walker, 1988; Keilmann and Michek, 1993; Miller and Schutte, 1993; Herzel and Reuter, 1997; Chuberre, 2000; Thurman et al., 2004;Svec et al., 2008; Roubeau et al., 2009). The physical nature of this transition is poorly understood. Several authors associate it with a switch to the highest vocal register (whistle, flageolet, flute, bell, small, pipe).

This whistle register is characterized by consistent acoustic features, such as a concentration of the acoustic power in the two first harmonics (Walker, 1988), reduced power around $3 \mathrm{kHz}$ and enhanced jitter (Keilmann and Michek, 1993). Perceptually, its voice quality is well recognized (Walker, 1988) and consistently described as fluty, although some other perceptual attributes can vary considerably with the singer's expertise. Untrained singers often sound squeaky or breathy with reduced intensity and reduced range of intensity (Thurman et al., 2004), whereas trained coloratura sopranos can produce very intense and "resonant" sounds (Thurman et al., 2004; Garnier et al., 2010). The biomechanical properties of the larynx in this high range are unknown. Some previous studies reported several significant differences in laryngeal behavior compared to laryngeal mechanism M2; this suggests that the whistle register may result from a third, distinct laryngeal mechanism M3 (Chuberre, 2000; Henrich, 2006; Roubeau et al., 2009): these studies measured significantly weaker levels of subglottal pressure (Miller and Schutte, 1993) and air flow (Walker, 1988 ) as well as high values of open quotient $(O Q)$ (Henrich, 2001; Svec et al., 2008; Roubeau et al., 2009). Glottal vibratory amplitude was reported as smaller than in laryngeal mechanism M2, sometimes without contact between the vocal folds (Rothenberg, 1988; Miller and Schutte, 1993; Svec et al., 2008; Tsai et al., 2008). As a consequence, glottal contact may be difficult to detect, and electroglottographic (EGG) signals are of very small amplitude (Miller and Schutte, 1993; Chuberre, 2000; Henrich, 2001; Roubeau et al., 2009). A constriction of the supraglottal and pharyngeal cavities has been reported (Shipp et al., 1988; Svec et al., 2008). This can make endoscopic exploration difficult or impossible.

Three theories have been proposed to explain the physical nature of the whistle register. A first acoustic theory proposes that the weakening of the vocal fold vibration at very high pitch does not correspond to a fundamentally different laryngeal mechanism from M2 but simply arises from a change in source-filter interaction (Miller and Schutte, 1993). Indeed, sopranos are known to adjust the frequency of their first vocal tract resonance $(R 1)$ close to the fundamental frequency $\left(f_{0}\right)$ from $\sim 500$ to $1000 \mathrm{~Hz}$ (C5-C6) (Joliveau et al., 2004b; Garnier et al., 2010; Henrich et al., 2011). Miller and Schutte (1993) showed that the weakening of vocal fold vibration at very high pitch coincides, for some singers, with the end of the $R 1: f_{0}$ resonance tuning. Rothenberg (1988) showed that the artificial lengthening of the vocal tract with a tube lowers the maximum pitch to which the two singers of that study can extend the $R 1: f_{0}$ tuning and also shifts down the laryngeal transition to the whistle register. However, recent results have shown that sopranos can still utilize resonance strategies over their highest range by tuning their second vocal tract resonance $(R 2)$ to $f_{0}$ once $f_{0}$ exceeds the possible range of $R 1$ (Garnier et al., 2010). Therefore the weaker vibration of the vocal folds at very high pitch might not necessarily be a consequence of the absence of tuning of a vocal tract resonance.

In a second "damping" theory, it is proposed that high frequencies are produced and controlled by adjusting the vibrating length of the vocal folds by means of varying degrees of compression of the arytenoids (Pressman and Kelenen, 1955; Van den Berg, 1963; Thurman et al., 2004; Titze and Hunter, 2004). Many authors report the absence of complete closure of the vocal folds at very high pitch (Thurman et al., 2004; Svec et al., 2008; Tsai et al., 2008). However, most of them observed a vibration of the whole length of the folds, including the posterior chink (Keilmann and Michek, 1993; Svec et al., 2008).

In a third aero-acoustic theory, it is suggested that vocal folds vibrate in a "vortex-induced" fashion similar to that of lip whistling (Berry et al., 1996; Herzel and Reuter, 1997). In lip whistling, the air flow is modulated by the oscillatory motion of an air jet through the constricted lips (Wilson et al., 1971). The modulation frequency, i.e., the pitch of the lip whistle, is controlled by the second resonance of the vocal tract, behaving as an upstream resonator. Somewhat similarly, extreme high vocal pitches are hypothesized to be produced by an oscillating jet at a narrowed glottis. Pitch may be controlled by the second resonance of the vocal tract, behaving in this situation as a downstream resonator. Further supporting this hypothesis are the only horizontal surface movements of the vocal folds (Keilmann and Michek, 1993) reported in the whistle register, the rotation of water on the folds observed at the same frequency as their oscillation (Berry et al., 1996) as well as the close proximity between $R 2$ and $f_{0}$ observed in singers able to sing above D6 (Garnier et al., 2010).

Further understanding of the physiological nature of the transitions in the high soprano range is needed. This study investigates the high range of 12 sopranos with different levels of expertise, to characterize how their glottal behaviors and their voice spectra vary with pitch and intensity, the frequencies at which fundamental changes occur and whether these transitions are influenced by vocal training. Interesting phenomena observed from the EGG signal are then investigated further through high-speed glottal video imaging of one of these singers. These observations, in conjunction with parallel measurements of vocal tract adjustments (Garnier et al., 2010), enable discussion of the physiological nature of the transitions in the high soprano range and how they relate to changes in resonance tuning.

\section{MATERIALS AND METHODS}

\section{A. Acoustic and EGG measurements on 12 singers}

\section{The subjects}

The subjects of the study were 12 sopranos, aged from 18 to 29 years and selected for their ability to produce high pitches. Subjects NE1 to NE4 were non-expert singers, AD1 to AD4 were advanced students, and PR1 to PR4 were 
professionals. Additional details are presented in Garnier et al. (2010).

\section{The protocol}

In a first part, singers were asked to produce at least three glissandi over their whole tessitura using the vowel [a]. They began at pitches around C4-E4.

In a second part, singers sustained a single note for $4 \mathrm{~s}$, again on the vowel [a]. They were asked to maintain constant pitch and loudness and to limit vibrato. Three tokens of each note were produced for measurement. They started on the pitch A4 and continued, on an ascending diatonic scale, to the highest pitch they could produce. For 5 of the 12 singers, the maximum pitch that could be sustained for more than a second was the same as the highest pitch that they could produce briefly at the end of the ascending glissando (see Fig. 1). For the others, the glissando briefly achieved pitches higher than those that could be sustained by one or two tones (for NE2, NE3, NE4, and PR2) or by three to six tones (for AD1, AD2, and AD3). Only eight singers could sustain notes above C6, and only five singers could sustain notes above E6. Four of the singers (NE1, AD3, AD4, and PR4) exhibited a pitch range around E5-G5 (from two tones to an octave), over which they were able to produce intentionally two very different voice qualities with distinct measured properties. In this paper, these qualities are called full head and fluty resonant, but the analysis uses their acoustic properties rather than subjective assessments or names. Over this range in which the two qualities overlap, these four singers produced three tokens of each note in each of the qualities.

In a third part, these four singers were asked to produce decrescendi on the vowel [a] for several notes in the range where the two different qualities were possible. The loudest part of the descrescendo, at its beginning, was produced with the full head quality. At least three examples of each note were recorded. One singer also produced crescendi in this overlap range.

\section{The measurements}

The audio signal was recorded with a $1 / 4$-in pressure microphone (Brüel and Kjær 4944-A), placed $30 \mathrm{~cm}$ away from the singers' lips, then amplified (conditioning amplifier Brüel and Kjær Nexus 2690) and digitized with 16-bit resolution at a rate of $44.1 \mathrm{kHz}$ using a FireWire audio interface (MOTU 828). The sound pressure level was calibrated using the $1 \mathrm{kHz}$ internal reference signal of the conditioning amplifier.

The EGG signal was simultaneously recorded with a two-channel electroglottograph (Glottal Enterprises EG2), using medical gel to improve electric contact between the skin and the electrodes. Electrodes were placed on both sides of the thyroid cartilage while the singer was singing in her comfortable middle range. The best placement of the electrodes was found by monitoring the EGG waveform with an oscilloscope. Medical tape was used on each electrode, instead of the usual Velcro neck strap, to prevent the electrodes from moving down throughout the experiment. No automatic gain control was used.
During the second part of the protocol, vocal-tract resonance frequencies were measured by broad-band excitation at the mouth, while audio and EGG signals were simultaneously recorded. These vocal-tract resonance measurements are presented in a companion article (Garnier et al., 2010) and will be referred to when examining how laryngeal transitions relate to resonance adjustments.

Using MATLAB software, the following four parameters were extracted from the EGG signal, using an $80 \mathrm{~ms}$ sliding rectangular window with no overlap:

(1) The fundamental frequency $\left(f_{0}\right)$, calculated using an autocorrelation method.

(2) The amplitude of the EGG signal.

(3) The glottal $O Q$, defined as the ratio between the duration of the glottal open phase and the fundamental period. $O Q$ was computed from the closing (positive) and opening (negative) peaks detected in the derivative of the EGG signal (DEGG) (Henrich et al., 2004).

(4) The contact speed quotient (Qcs), defined as the ratio in amplitude of closing and opening peaks of the DEGG signal. This quotient describes the relative difference in speed of contact between glottal opening and closing. It reflects the degree of asymmetry of the EGG waveform.

The calibrated sound pressure level (SPL) and voice spectrum were measured from the audio signal, using an 80 ms sliding rectangular window with no overlap. The levels of voice harmonics $\left(A H_{\mathrm{i}}\right.$, in $\left.\mathrm{dB}\right)$ were then extracted from the voice spectrum to estimate $r=$ mean $\left(A H_{1}, A H_{2}\right)-$ mean $\left(A H_{3}, A H_{\mathrm{i} \leq 10 \mathrm{kHz}}\right)$, a parameter quantifying the difference between the average level of the two first harmonics and that of the other harmonics below $10 \mathrm{kHz}$.

For the sustained pitches of the second session, the mean value of glottal parameters was computed over the $4 \mathrm{~s}$ of phonation, whereas the mean SPL and mean $r$ value were computed only from the first second of the audio signal before the vocal tract was excited by the external broadband signal.

Previous studies showed how, on glissandi, the change in laryngeal mechanism from M1 to M2 is characterized by a sudden decrease in glottal contact (translating into a decrease in amplitude of the EGG signal), a sudden increase in $O Q$ and a sudden change toward more symmetrical waveform of the EGG signal (Roubeau et al., 1987, 1991; Henrich, 2001; Henrich et al., 2005; Roubeau et al., 2009).

In the glissandi produced in this study, we verified that these sudden changes in the EGG signal, typical of the M1-M2 transition (the primo passaggio), and often accompanied by a pitch jump, always occurred below E4. Consequently, sopranos were always using mechanism M2 in their middle range ( $\sim \mathrm{F} 4-\mathrm{G} 4)$, corresponding to the bottom of the range considered for analysis in this paper and its companion study (Garnier et al., 2010). It follows that the transitions above F4 described in this article, and especially those far above F4, cannot be interpreted as the M1-M2 transition.

\section{B. Endoscopic investigation on one singer}

Singer NE1 undertook further endoscopic investigations of glissandi and decrescendi in her high-pitch range, to 
relate features of the EGG signal in this range to visual observations of the glottis. Endoscopy was not available for the other 11 singers.

High-speed video recording of the vocal fold vibration was carried out at the Georges Pompidou European Hospital (HEGP) in Paris using a high speed camera (Weinberger SL Kamera 2000) connected to a SpeedCam + Lite acquisition system. Two thousand frames were recorded per second with a resolution of $256 \times 256$ pixels. The EGG signal (Portable Laryngograph) was recorded in synchrony with the audio signal (AKG C410 microphone) on the computer. Both signals were preamplified with a Diana interface (SQLab).

\section{RESULTS}

Figure 1 indicates the pitch range explored for each soprano, from F4 (above the M1-M2 transition) to the upper limit of her vocal range. It summarizes different information on vocal transitions in this high range: (1) variations in amplitude of the EGG signal, (2) regions of pitch jumps or voice instabilities, and (3) ranges where different resonance tuning strategies were used. In this figure (as well as in Figs. 2 and 3), data for each token on each subject are included to indicate intra-subject variability.

\section{A. Changes and transitions observed during glissandi}

\section{A laryngeal transition characterized by a decrease in EGG amplitude}

The vertical axis in Fig. 1 is the amplitude of the EGG signal. This was always weaker at the top of the singers' vocal range than in the medium range $(\sim F 4)$. This decrease in amplitude may be interpreted as a decrease in glottal contact. Another possible contributing factor is the rising larynx that most sopranos reported feeling at high pitch and described in several studies (Shipp and Izdebski, 1975; Sonninen et al., 1999). In any case, this decrease in amplitude indicates a transition between two stable laryngeal behaviors. The lower and upper limits of the laryngeal transition respectively were identified by visual inspection as the pitches immediately before and after the decrease in amplitude. The pitch range of the transition was the difference between the upper and lower limits.

On average, the amplitude of the EGG signal was reduced by a factor of $4.2 \pm 1.5$ during this transition (for comparison, Roubeau et al., 1987 reported a decrease by only 1.8 during the M1-M2 transition for women.)

Although this laryngeal transition always occurred at pitches above those of the M1-M2 transition, it could start as low as G4 (for singers NE3, NE4, and PR4) or as high as D5-D\#5 (for singers AD4 and PR1), the average starting pitch being A\#4 \pm 3 semitones. Expertise did not appear to influence the lower pitch limit of the transition. The upper pitch limit of the transition also varied considerably among singers: non-expert singers tended to complete the laryngeal transition at lower pitches ( D D 5) than advanced and professional singers (from F\#5 to D\#6), except for singers NE2 and AD3. The laryngeal transition sometimes occurred very rapidly with increasing pitch (e.g., NE1) or could be spread over an octave (e.g., PR4).

In most cases, there were one or more pitches in the transition range for which no variation in glottal contact was
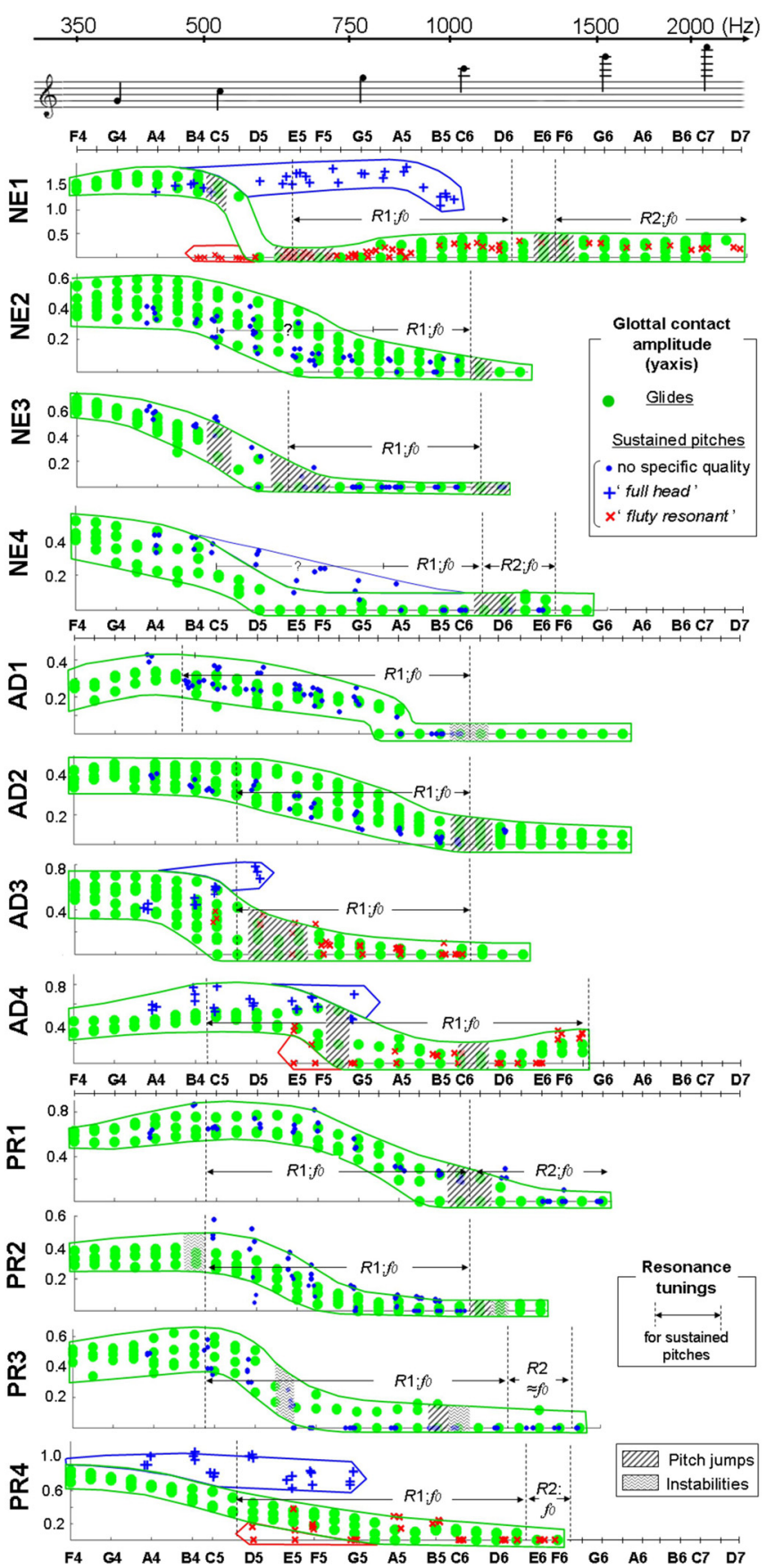

FIG. 1. (Color online) Summary of the observations on the 12 singers. Large pale circles represent, in the arbitrary units of the electroglottograph (no automatic gain control was used), the variation in glottal contact amplitude with increasing pitch on glissandi. (The multiple points at each pitch are from different glissandi and indicate the reproducibility.) Small dark dots represent this variation for sustained pitches produced without any specific intended quality, while dark + and $\times$ symbols represent this variation for sustained pitches produced with, respectively, a full head or a fluty resonant intended quality. Dashed or waved areas indicate pitch ranges where pitch jumps and instabilities were observed on glissandi. Horizontal arrows indicate the ranges of sustained notes over which vocal tract resonances were tuned to the first voice harmonic $\left(f_{0}\right)$ (reported from Garnier et al., 2010). 
detected by EGG, i.e., the EGG signal showed no periodic waveform. In a few cases, however (on some of the glissandi produced by singers NE2, AD2, PR1 to PR4), glottal contact variation was maintained throughout the transition. For 10 of the 12 singers, a small-amplitude, periodic EGG signal could be detected above the transition. No substantial change in the amplitude of the EGG signal was then observed with increasing pitch (see Fig. 1).

\section{Variations in open quotient (OQ)}

In Figs. 2 and 3, several acoustic and glottal parameters are plotted as functions of pitch for four singers to illustrate different behaviors observed. Two contrasting behaviors were observed in the $O Q$ :

In 10 singers (PR2-4, AD1,3-4, NE1-4), $O Q$ increased smoothly with increasing $f_{0}$ over the pitch range throughout which the amplitude of the EGG signal decreased [see Figs. 2(b) and 3]. However, the parameters did not always vary in complete synchrony: For different singers, $O Q$ started increasing at pitches that were slightly higher or lower than those of the decrease in contact amplitude. The maximum values of $O Q$, higher than 0.8 and mostly around 0.9 , were reached at the upper limit of the laryngeal transition. Above the transition, $O Q$ tended to decrease continuously with ascending pitch for seven singers [see Figs. 2(b) and 3] and remained constant for one (singer AD3).

For two singers (PR1, AD2), $O Q$ decreased with $f_{0}$ over the first part of the transition interval [see glissandi chart of Fig. 2(a)]. It then reached a local minimum four semitones before the amplitude of the EGG signal reached its lowest level and then continuously increased with pitch over the remaining transition interval and above.

\section{Variation in contact speed quotient (Qcs)}

The contact speed quotient consistently decreased smoothly with ascending $f_{0}$ for 10 of the singers over the pitch range in which the amplitude of the EGG signal decreased (see Fig. 2), indicating that the EGG waveform was becoming more symmetrical. The variation in $Q c s$ was not completely synchronous with the variation in amplitude of the EGG signal or with the variation in $O Q$. Above the laryngeal transition, no significant change in Qcs was observed with increasing $f_{0}$ (see Figs. 2 and 3). For two singers (NE2 and PR1), Qcs continued to decrease slightly with increasing $f_{0}$.

\section{Variations in the EGG waveform}

Apart from NE1, for whom the laryngeal transition was abrupt, no singer showed any discontinuous variation in laryngeal parameters during the transition. As a consequence, the EGG signal presented intermediate waveforms between the typical M2 waveform observed below the transition and the EGG waveform observed above it (see Fig. 4).

\section{Variation in SPL}

For most singers, SPL was typically 10-15 dB greater above the laryngeal transition than below. For some singers
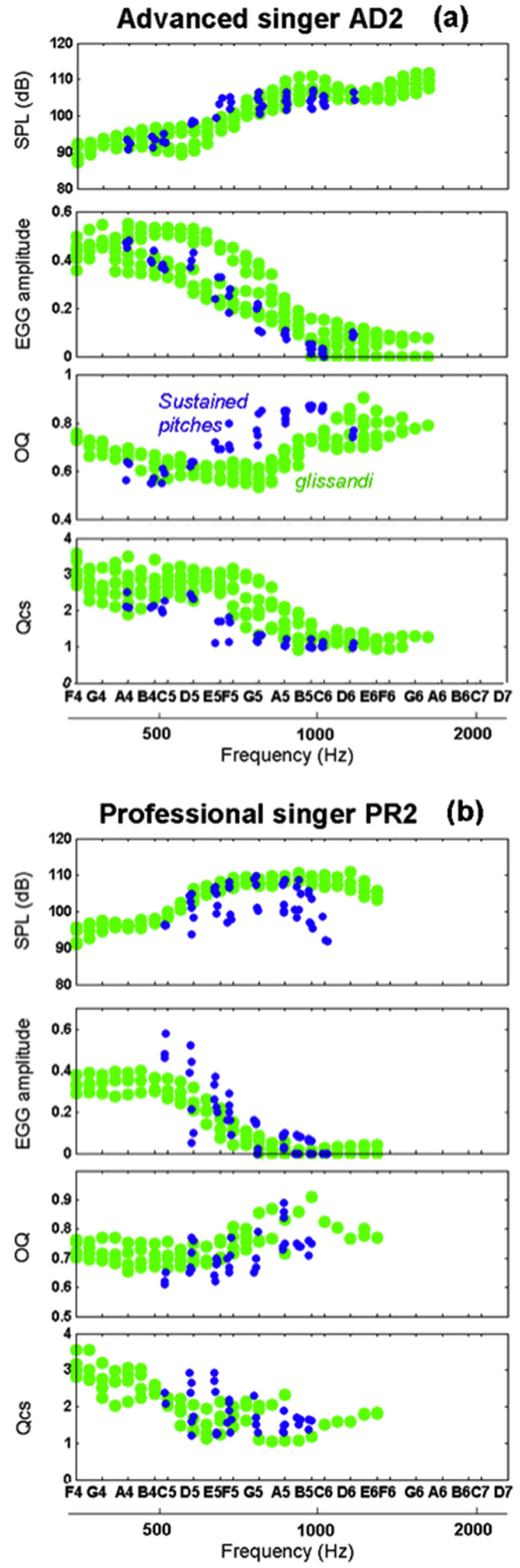

FIG. 2. (Color online) The variation of sound pressure level (SPL), amplitude of the EGG signal, open quotient $(O Q)$, and the contact speed quotient $(Q c s)$ with increasing pitch for the advanced singer AD2 (a) and the professional singer PR2 (b), measured on continuous glissandi (light grey) or sustained pitches (dark). 
Professional singer PR4 (a)
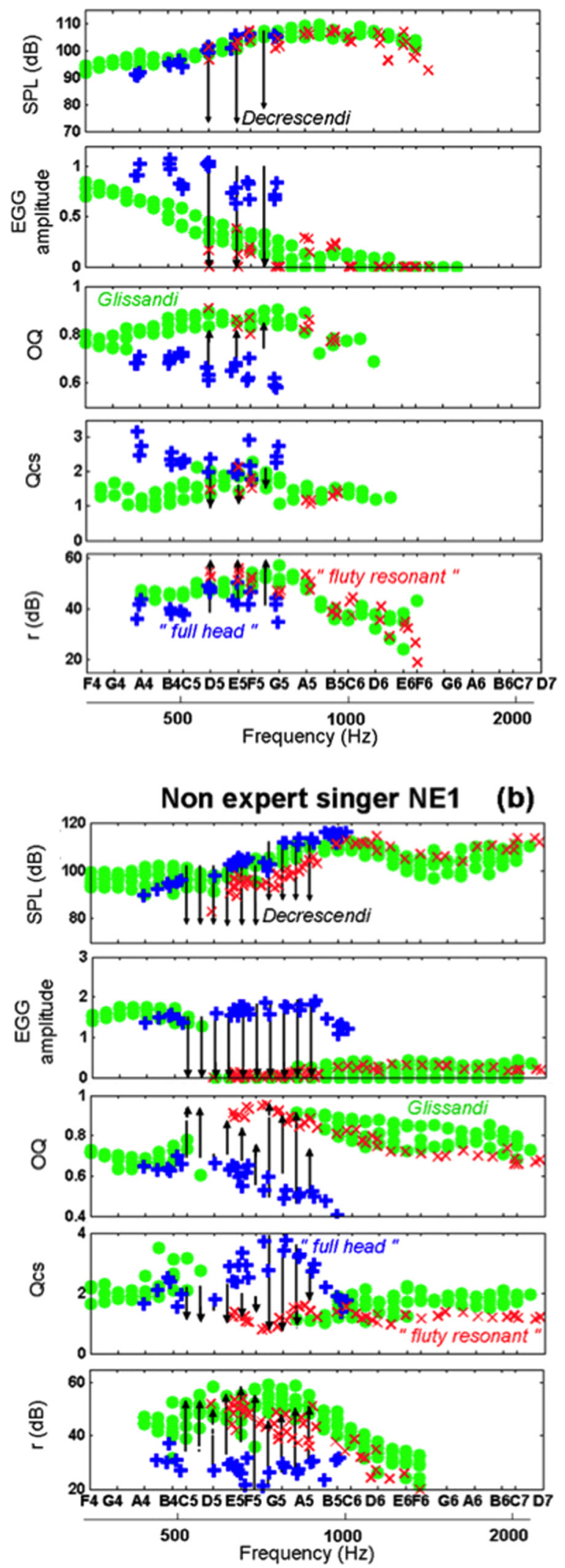

FIG. 3. (Color online) The variation of SPL, amplitude of the EGG signal, open quotient $(O Q)$, contact speed quotient $(Q c s)$, and enhancement of the two first voice harmonics $(r)$ with increasing pitch for the professional singer PR4 (a) and the nonexpert singer NE1 (b). They were measured on continuous glissandi (big pale dots), on sustained pitches in full head quality $($ dark bold + ) or in fluty resonant quality $(\times$ symbols), and on continuous decrescendi (black lines).

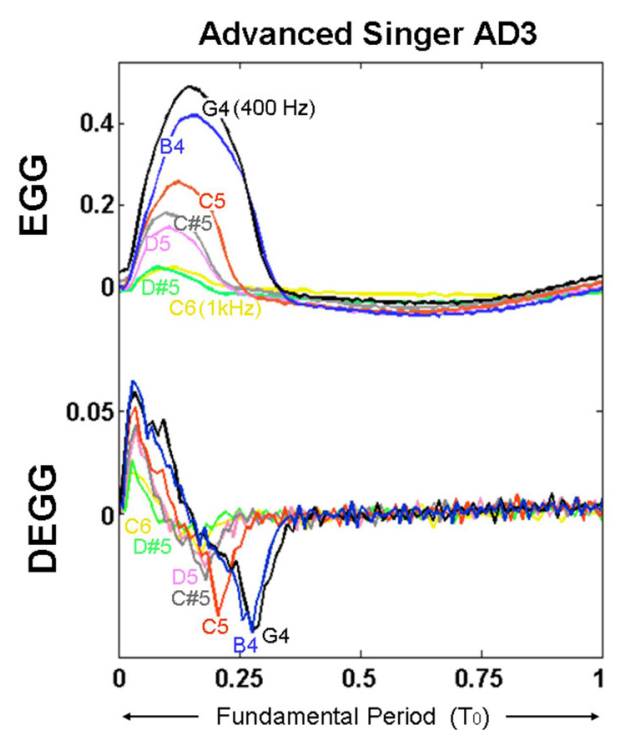

FIG. 4. (Color online) Waveforms of the EGG and DEGG signal measured for singer $\mathrm{AD} 3$ before, during, and after her laryngeal transition, which occurred between B4 and D\#5.

(NE2, AD1, AD2, PR1-PR4), it increased during the transition and reached its maximum value at the end of it [see Figs. 2 and 3(a)]. For other singers, it started increasing around C5-D5, which was either above (AD3, NE1, NE3 and NE4) or below (AD4) the starting pitch of the transition [see Fig. 3(b)].

For all singers, SPL reached its maximum value in the range A5-C6 and then decreased at higher pitch. For some singers, the decrease was small [see Fig. 3(a)]. For others, the decrease could be as much as $10 \mathrm{~dB}$ between $\mathrm{C} 6$ and G6 [see Fig. 3(b)]. Singers who sang above E6 demonstrated a second increase of SPL with $f_{0}$ over their top range where no significant change was observed in glottal parameters [see Figs. 2(a) and 3(b)].

\section{Pitch breaks, pitch jumps, and instabilities}

Although singers were instructed not to avoid breaks, the professional and advanced singers exhibited fewer pitch breaks or jumps than did non-expert singers. A reason may be that part of vocal training consists in learning how to smooth and hide register transitions. Therefore, voice instabilities during the glissandi were considered in addition to pitch breaks or jumps. They were quantified by computing $\mathrm{d} f_{0} / \mathrm{dt}$ as a function of $f_{0}$ and identifying local extrema.

Figure 1 summarizes for each singer the pitch range(s) over which pitch jumps (striped shading) or instabilities (wavy shading) were observed during the different glissandi. There were three main pitch ranges where instability occurred:

(1) around B4-C5. This concerned only three singers. Systematic jumps were observed for two non expert singers (NE1 and NE3) and the professional singer PR2 exhibited consistent instability.

(2) around E5 (from D5 to F\#5 depending on the singer). This concerned five singers: non-experts (NE1, NE3), 
advanced and professional singers (AD3, AD4, and PR3).

(3) around C\#6 (from B5 to E6 depending on the singer). Ten of the 12 singers showed pitch jumps or instability in this region.

One singer (PR4) exhibited no pitch breaks, jumps, or instabilities in the pitch range studied. Half of the singers (NE2, NE4, AD1, AD2, AD3, and PR1) exhibited instability in only one pitch range, which coincided with the upper limit of the M2-M3 laryngeal transition (i.e., the pitch from which the amplitude of the EGG signal stopped decreasing). This upper limit varied among singers, observed as low as D5 and as high as D6 (Fig. 1). Three singers (AD4, PR2, and PR3) showed instabilities in two pitch ranges, and two singers (NE1 and NE3) exhibited instabilities in three pitch ranges. In four of these five singers, the instabilities also occurred at the upper limit of the laryngeal transition.

Taking all these cases together, 10 of the 12 singers exhibited a region of pitch instability near the upper limit of the laryngeal transition, around D5-F\#5 (for NE1, AD3, AD4, and PR3) or around C6-D6 (for NE2, NE3, NE4, AD1, AD2, and PR1). Singers PR2 and PR4 behaved differently from the other singers: PR4 presented no instabilities at all over her high range, and PR2 exhibited jumps at C6, far above her laryngeal transition pitch range.

Consequently, it appears to be more relevant to relate voice instabilities to ranges or events in the glottal behavior instead of considering only the pitch at which they occur. As mentioned in the above text, they commonly occurred at the upper limit of the laryngeal transition (for 10 singers of 12). They sometimes occurred below the transition, when the amplitude of the EGG signal started decreasing (around B4-C5 for NE1 and PR2). They often also occurred far above the laryngeal transition, in a range (from $\mathrm{C \# 6}$ to F6) where the glottal behavior showed no significant change (NE1, NE3, AD4, PR2, and PR3).

\section{Direct endoscopic observations}

A descending glissando from D7 $(2350 \mathrm{~Hz})$ to E4 $(330 \mathrm{~Hz})$ produced by singer NE1 was analyzed with highspeed video, audio, and EGG recordings, as illustrated in Fig. 5. It presented several pitch jumps and changes in voice quality.

A glottal contact was detected on the upper pitch range of the glide, down to D\#6. A small pitch jump was observed near F6 corresponding to no particular change in vibration pattern nor in the EGG waveform. A stretched glottis could be seen on the high-speed images. At these high pitches, the glottal cycle could be characterized with a maximum of two images, which showed either a vocal fold contact or a smallamplitude opening (see the two images taken near $1.65 \mathrm{~s}$ and presented at the bottom left of Fig. 5).

Between D\#6 and G5, no glottal contact could be detected by EGG. This range corresponded to constriction in the epilaryngeal tube, clearly noticeable on the high-speed sequence. The maximum constriction coincided with an abrupt pitch decrease recorded in the audio signal at $2.2 \mathrm{~s}$.

Below G5, glottal contact was again detected and its amplitude suddenly increased around E5 together with a pitch jump.

\section{B. Sustained pitches and changes in voice quality}

For six singers (PR3, PR2, AD1, NE2, NE3, NE4), the behavior of the glottal parameters was the same for sustained pitches as for glissandi [see Fig. 2(b)].

For two singers, however, some differences were observed between glissandi and sustained pitches. Interestingly, these two singers were AD2 and PR1, whose variations of $O Q$ differed from those of the other singers during glissandi. For AD2, the variation of $O Q$ on sustained pitches became similar to that observed in most of the singers: It increased with pitch over the laryngeal transition, i.e., the pitch range where the amplitude of the EGG decreases [see Fig. 2(a)].

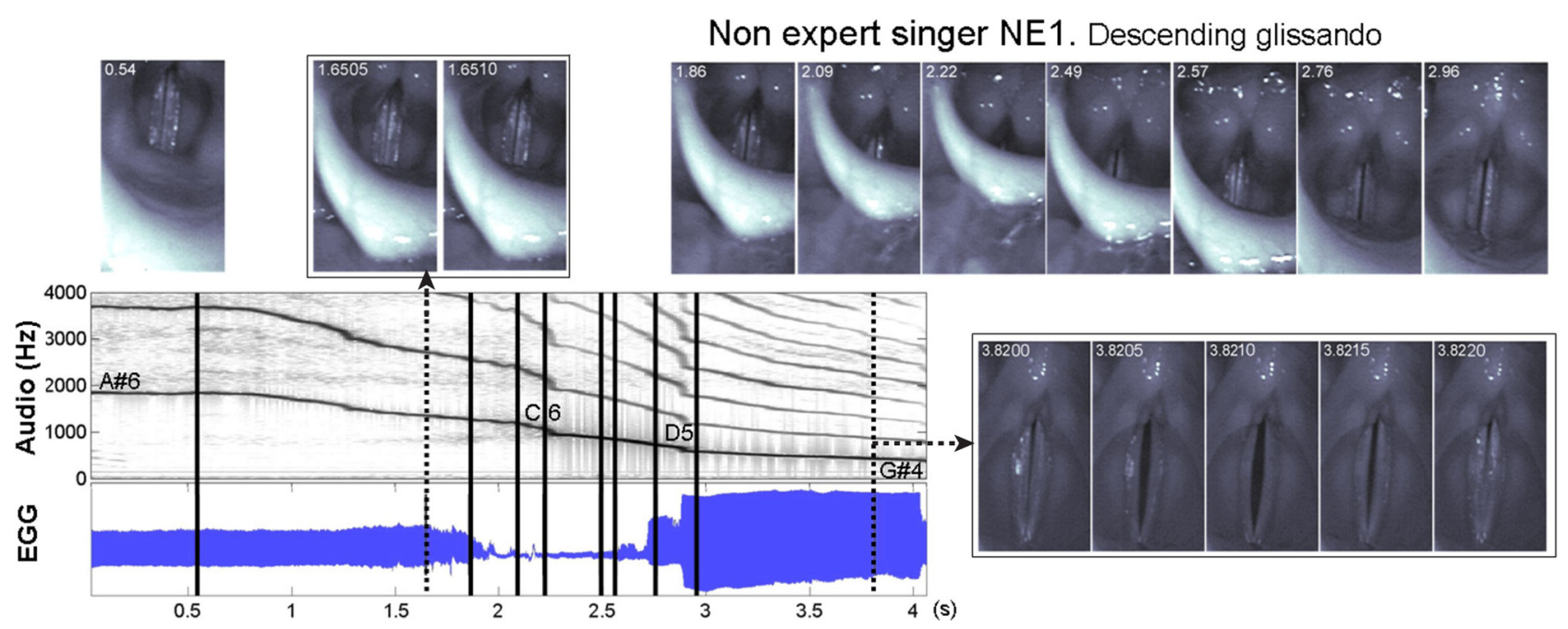

FIG. 5. (Color online) A descending glissando produced by singer NE1. Vertical lines on the spectrographic sound analysis indicate the corresponding instants of the endoscopic images. Two glottal-cycle sequences (dashed lines) illustrate the stable behavior observed before and after the laryngeal transition (high pitch, two images; lower pitch, five images). 
The four remaining singers (PR4, AD3, AD4, NE1) showed an overlap range over which they could produce two different qualities with distinctly different EGG signals, referred to here as full head and fluty resonant. For all these four singers, the comparison of sustained pitches produced in both qualities showed clear and consistent differences in glottal parameters: The EGG amplitude and the contact speed quotient $(Q c s)$ were significantly lower in the fluty resonant quality than in the full head one, whereas $O Q$ values were significantly higher in the fluty resonant quality than in the full head one (see Fig. 3). Comparison of sustained pitches produced in both qualities showed clear and consistent differences in spectral content and SPL for two of the four singers (NE1 and AD4): Their fluty resonant quality tended to be $10 \mathrm{~dB}$ weaker than the full head one, with the higher harmonics being especially weak with respect to the first two [see Fig. 3(b)]. For the two other singers (PR4 and AD3), the two qualities were produced with similar SPL values [see Fig. 3(a)]. Harmonics above the first two were also weak in the fluty resonant quality, but to a lesser extent than for NE1 and AD4.

Last, the values of glottal parameters $O Q$ and $Q c s$ in full head sustained productions (see $\times$ symbols on Figs. 1 and 3 ) were similar to those observed on glissandi (see pale dots in Figs. 1 and 3 ) prior to the laryngeal transition, i.e., before the amplitude of the EGG signal starts decreasing. On the other hand, values in fluty resonant sustained productions

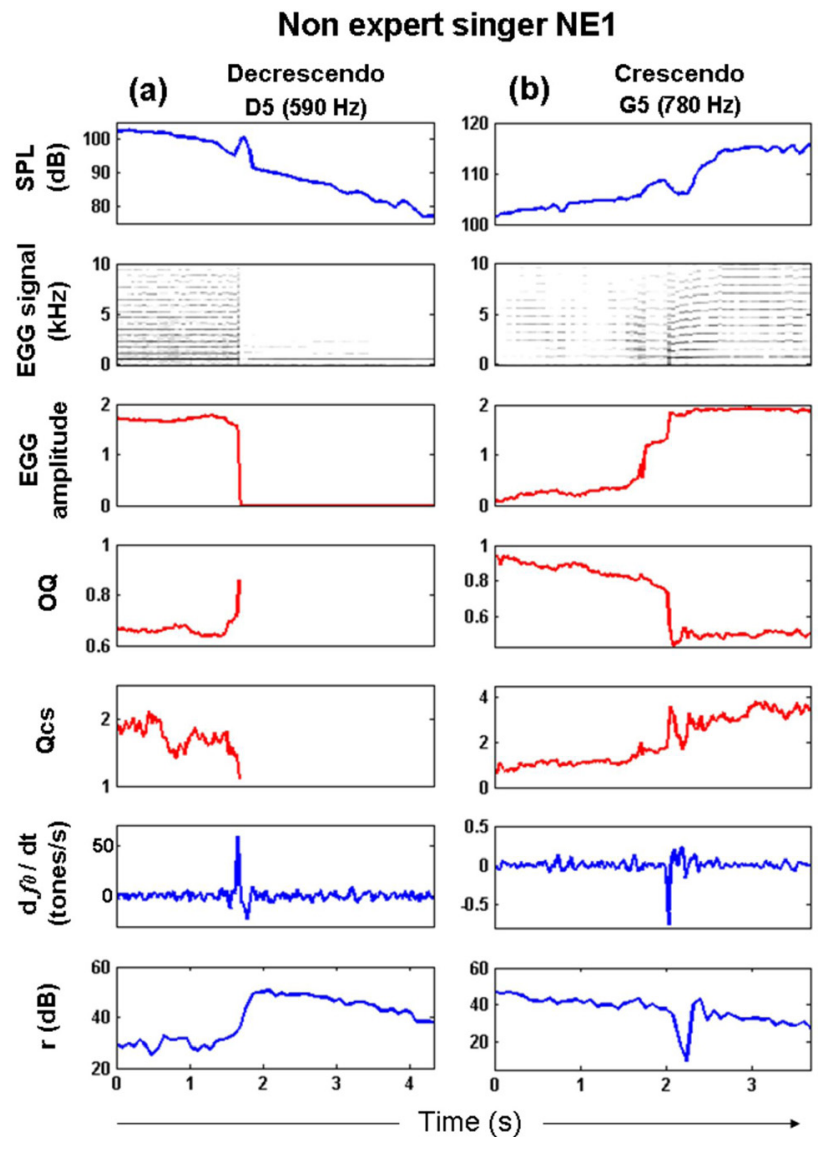

FIG. 6. (Color online) Example of the variation of measured acoustic and glottal parameters during a decrescendo and crescendo produced on D5 and G5 respectively by singer NE1. (see + symbols in Figs. 1 and 3) were similar to those observed on glissandi above the transition, i.e., after the amplitude of the EGG signal had reached its lower level.

Consequently, it appears that for these singers, full head quality is produced in M2 within the overlap range (M2-M3 transition shifted higher in pitch), whereas fluty resonant quality is produced in M3 instead (M2-M3 transition lowered).

\section{Decrescendi}

The four singers who demonstrated an overlap range of the two glottal behaviors were asked to produce decrescendi on a few sustained notes within that range.

An abrupt decrease in the amplitude of the EGG signal was observed, with continuously decreasing SPL, followed by the disappearance of any observable EGG waveform [see second panel in Fig. 6(a)].

For NE1, AD3, and PR4, this sudden decrease in amplitude was systematically accompanied by pitch jumps [see $\mathrm{d} f_{0}(\mathrm{t}) / \mathrm{dt}$ in Fig. 6(a)], whereas jumps occurred only in some
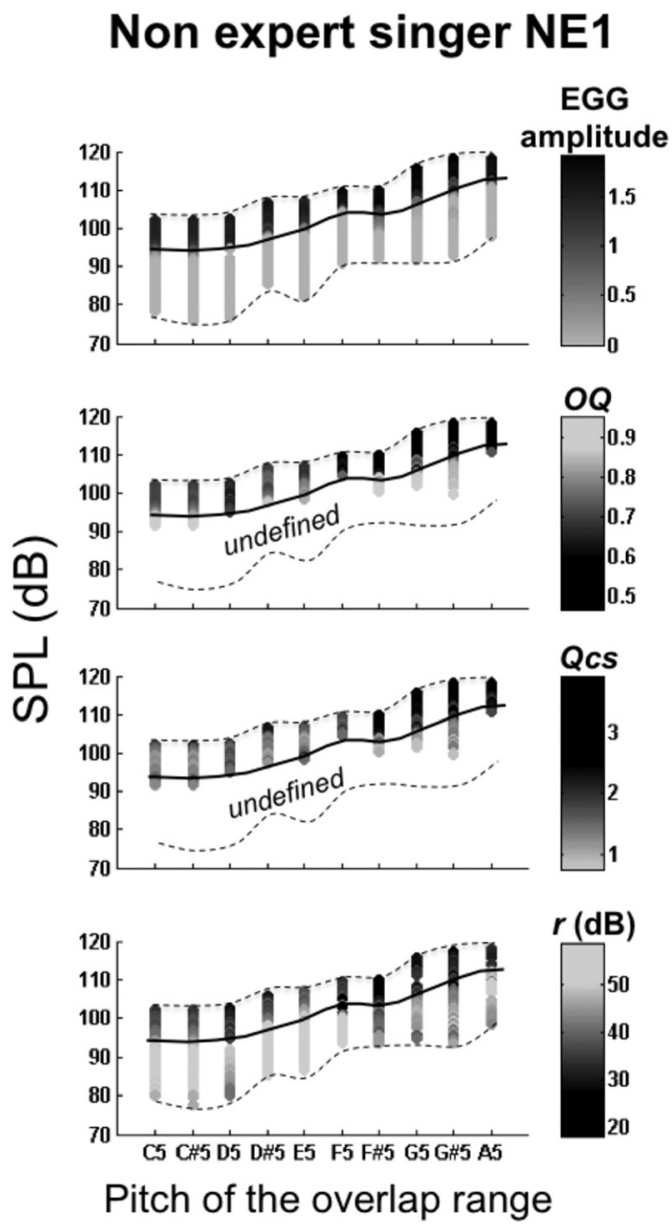

FIG. 7. The variation of amplitude of the EGG signal, open quotient $(O Q)$, contact speed quotient $(Q c s)$, and enhancement of the two first voice harmonics $(r)$ with decreasing SPL and several pitches of the overlap range for the non expert singer NE1. The dotted lines indicate the limits of the SPL range explored for each decrescendo. The solid line indicates, for each pitch, the SPL from which the amplitude of the EGG signal suddenly decreased and became null. In that range where no glottal contact was observed any longer, the glottal parameters OQ and Qcs could consequently not be defined. 
Non expert singer NE1. Decrescendo F5-F\#5 $(700 \mathrm{~Hz})$

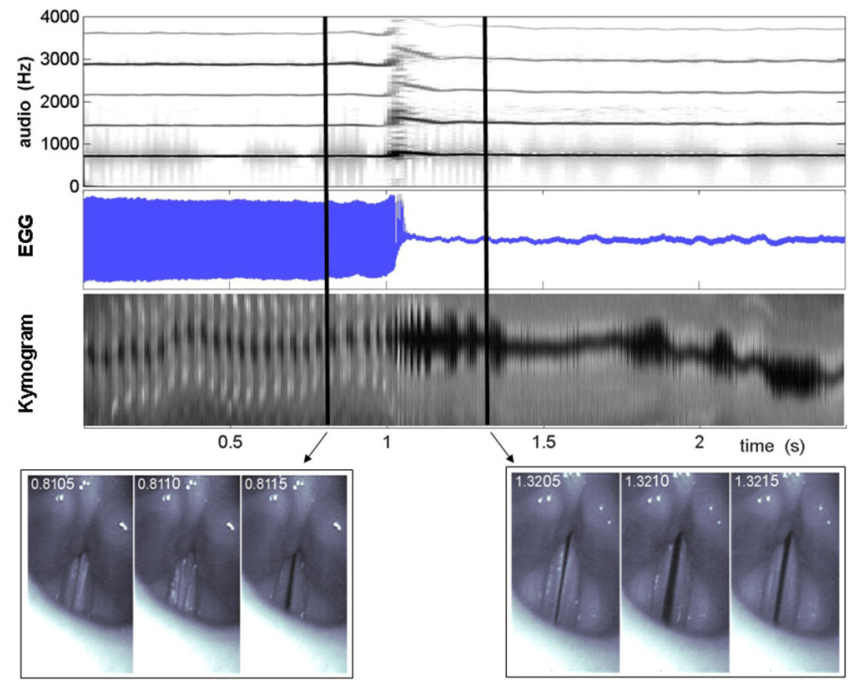

FIG. 8. (Color online) A decrescendo produced by singer NE1 on pitch F5-F5\#. Top panel: spectrographic sound analysis. Middle upper panel: EGG signal. The vertical dark lines indicate the shot instants. Middle lower panel: kymographic analysis of a median line. Bottom panel: two glottal-cycle sequences illustrating the stable behavior before and after the pitch break.

cases for AD4. Pitch jumps were greater for decrescendi on higher notes.

For all four singers, the sudden decrease in amplitude of the EGG signal was also accompanied by a sudden decrease in the proportion of acoustic power in voice harmonics above the first two [see lowest panel of Figs. 6(a) and 7]. This explains the sudden change in perceived voice quality, from full head at high SPL to fluty resonant at lower SPL.

As was observed for the glissandi over the laryngeal transition, $O Q$ increased over the SPL range for which the EGG signal decreased, whereas Qcs tended to decrease (see Qcs in Figs. 6 and 7).

Values of glottal and acoustic parameters at the beginning of the decrescendo, i.e., at high SPL, are comparable with those measured on glissandi around G4-A4, below the laryngeal transition and with those measured on full head sustained pitches over the overlap range. On the other hand, values of glottal and acoustic parameters measured at lower SPL of the decrescendi, after the amplitude of the EGG signal decreased (and just before it disappeared for $O Q$ and $Q c s$ ), are comparable with those measured on glissandi around A5-B5, above the laryngeal transition and with those measured on fluty resonant sustained pitches over the overlap range (see Fig. 3).

Comparison of decrescendi produced on different pitches within the overlap range of the four singers, indicates that the transition in glottal behavior and in voice quality occurred at higher SPL when the pitch increased (see Fig. 7).

In a separate session, singer NE1 produced crescendi over the overlap range of the two glottal behaviors. Observations were consistent with those made on decrescendi: in crescendi, the amplitude of the EGG signal increased suddenly with increasing SPL, accompanied by pitch jumps and sudden variation in $O Q, Q c s$, and temporary enhancement of the voice harmonics above the first two [see Fig. 6(b)]. The transition in glottal behavior did not occur at lower SPL on crescendi than on decrescendi. Thus for this laryngeal transition and for this singer, there did not appear to be any hysteresis of the sort reported for the M1-M2 transition (Roubeau et al., 2004). As a consequence, NE1 did not show any overlap range in SPL within which she could choose, for a given pitch, to use one glottal behavior and its associated voice quality instead of the other.

The glottal behavior of singer NE1 on a decrescendo on pitch F5-F5\# $(700 \mathrm{~Hz})$ that produced a pitch break, and a change in voice quality was assessed with combined highspeed laryngoscopy, audio, and EGG. Figure 8 plots the spectrographic analysis, the EGG signal, a kymogram of a median line, and the images from two glottal cycles within the sequence. Voice quality went from full head to fluty resonant. The spectrographic analysis and the EGG signal showed a noticeable laryngeal transition, with a pitch jump (F5 to F5\#) and a brief subharmonic pattern (about $30 \mathrm{~ms}$ ). The kymographic analysis and the glottal images showed no glottal contact after the pitch break nor did the EGG signal (no contact detected after $1.1 \mathrm{~s}$ ).

\section{DISCUSSION}

\section{A. The laryngeal nature of the whistle register}

The EGG and direct endoscopic investigations suggest that there is only one main laryngeal transition in the high soprano range that occurs above the M1-M2 transition or primo passaggio.

Some similarities between the laryngeal transition reported here and the $\mathrm{M} 1-\mathrm{M} 2$ transition support the idea that the higher transition corresponds to the fundamental change between laryngeal mechanisms M2 and M3 proposed by Roubeau et al. (2009). Indeed, as is observed for the M1-M2 transition (Henrich, 2001; Henrich et al., 2005; Roubeau et al., 2009), the amplitude of the EGG signal decreased significantly with ascending pitch during the M2-M3 transition, while $O Q$ increased and the EGG waveform became more symmetrical. The M2-M3 transition was accompanied by a substantial change in voice spectrum, with reduced energy in harmonics above the first two producing a flutier quality. Similarly to the M1-M2 transition, these results show that there can be an overlap pitch range for the two M2 and M3 glottal behaviors observed below and above the transition. This overlap range was observed around E5 and could cover up to one octave (C5-C6), which corresponds to the typical range of the upper register (Miller, D.G., 2000; Miller, R., 2000; Echternach et al., 2010). Similarly to the M1-M2 overlap range, decrescendi produced over the overlap range of M2 and M3 showed a sudden change from one glottal behavior to the other, accompanied by pitch jumps or voice instabilities at the transition as well as noticeable changes in voice spectrum.

However, the M2-M3 transition differs from the M1-M2 in several aspects; this rather supports the idea that while the M2-M3 laryngeal transition corresponds to a change in glottal behavior, that change is not always an 
abrupt and fundamental change in vocal fold biomechanics like that observed at the M1-M2 transition. Indeed minor modifications in vocal fold amplitude of vibration and contact were observed before and after the transition on the high-speed video of one singer. An epilaryngeal constrictive movement was observed at the top of the M2 range released when transitioning to the M3 laryngeal behavior. Furthermore, in the EGG signals, continuous transition from M2 to M3 behavior was often observed to occur over a range of several notes, which is not the case for M1-M2 laryngeal transition.

Further electromyographic, aerodynamic and endoscopic data would be needed to understand better the biomechanical principles of this main change in laryngeal behavior in the high soprano range.

\section{B. Whistle register and resonance adjustments}

As reviewed in the Introduction, previous studies have supported the idea that the transition to whistle register is caused by a change in the acoustic load on the vocal folds produced by changes in vocal tract resonance (Miller and Schutte 1993; Rothenberg 1988). In this study, however, only few singers showed a correspondence among (1) pitch jumps or instabilities, (2) the end of $R 1: f_{0}$ tuning (presented in the companion paper Garnier et al., 2010), and (3) the end of the M2-M3 laryngeal transition. For most singers, the change in glottal behavior and voice quality occurred around C5-E5, far below the upper limit of the $R 1: f_{0}$ tuning (around C6-D6). It was also observed that some singers can extend the $R 1: f_{0}$ tuning up to $\mathrm{F} \# 6$, whereas they always changed glottal behavior and voice quality below C6 (Garnier et al., 2010). Because the M2-M3 laryngeal transition coincides with the upper limit of a regime of resonance tuning for only a small proportion of singers, it appears that this laryngeal transition is not caused by the end of $R 1: f_{0}$ resonance tuning. The physiological reasons for this change in laryngeal behavior remain unidentified.

\section{Pitch jumps and breaks in the high soprano range}

Additional pitch jumps or instabilities occurred over the high soprano range at pitches other than the end of the M2M3 laryngeal transition. In particular, singers who demonstrate the M3 behavior from as low as D5-E5 can also have pitch jumps or instabilities around C6-D6, although no noticeable change was observed in their glottal behavior in that range. The pitch range C6-D6 corresponds to the range from which singers start tuning $R 2$ to $f_{0}$ instead of $R 1$ (Garnier et al., 2010) and from which the SPL starts increasing again. The pitch jumps and instabilities observed around these pitches are very likely to be induced by acoustical interactions between the tract and the glottal source.

\section{Implications for voice quality and voice classification}

Because the starting pitch of the M3 behavior is not caused by the limits of a vocal tract tuning regime, some singers demonstrated the ability to lower or raise the
M2-M3 transition to some extent, similarly to the transition pitch of M1 and M2 (Roubeau et al., 2004; Henrich, 2006; Roubeau et al., 2009). Consequently, there can be an overlap range (as much as one octave in this study) over which singers can choose to use M2 or M3 depending on the intended voice quality.

The qualities described here as fluty resonant (with reduced glottal contact) and full head quality (with M2 vibratory pattern) were not associated with different strategies of resonance tuning. Despite reduced glottal contact, sounds produced with the M3 behavior were often resonant and rarely breathy. They could be very loud: up to $115 \mathrm{~dB}$ SPL at $30 \mathrm{~cm}$. Furthermore, our results indicate that sopranos, irrespective of their vocal expertise, can start using the laryngeal mechanism M3 from as low as C5-D5, which is one octave lower than what is usually reported for the whistle register (Van Deinse, 1981; Walker, 1988; Miller and Schutte, 1993; Herzel and Reuter, 1997; Thurman et al., 2004; Henrich, 2006). Consequently, the mechanism M3 should not be considered as a marginal type of voice production found only in a few singers at extreme pitches.

These results suggest that light, soubrette, or coloratura sopranos - those who demonstrate a fluty sound above E5 and who are able to produce extreme pitches with agility-might correspond to the category of singers who use the M3 mechanism from as low as D5-E5. On the other hand, sopranos classified as full lyric or dramatic-those who demonstrate a full and rich sound above E5-are likely to correspond to the category of singers who use the M2 mechanism in their upper register. These considerations imply that light or coloratura sopranos might benefit from slightly different training methods than lyric or dramatic sopranos.

\section{E. Voice registers and transitions in the high soprano range}

A singing voice register can be considered as a distinct region of homogeneous voice quality that can be maintained over some ranges of pitch and loudness (Titze, 1994). Voice quality depends not only on the laryngeal mechanism but also on the vocal tract configuration and its influence on vocal fold vibration.

D. G. Miller (2000) suggested that the female voice is divided, above the M1-M2 transition, into three registers: middle, upper, and flageolet registers, all underlined by similar falsetto $(\sim \mathrm{M} 2)$ pattern of vocal fold vibration, and differing in vocal tract adjustment.

The present study and its companion paper support that proposed division: two main changes in vocal tract adjustment were observed in the high soprano range. One indicates the lower limit of $R 1: f_{0}$ tuning, which, for some singers and vowels, starts in the vicinity of C5 (Joliveau et al., 2004a,b; Garnier et al., 2010; Henrich et al., 2011), i.e., the typical region of the middle/upper transition (Miller, D.G, 2000). The second, which occurs typically around C6, is the upper limit of $R 1: f_{0}$ tuning (Garnier et al., 2010).

This second resonance transition did not always coincide with a weakening of vocal fold vibration, in contrast 
with the observation of D. G. Miller (2000) and Rothenberg (1988). In the present study, there was always a finite transition interval over which the amplitude of the EGG signal decreased significantly. However, the low-amplitude EGG waveform (corresponding to the M3 glottal behavior) was often reached below the upper limit of $R 1: f_{0}$ tuning. Furthermore, for singers who could sing above the end of the $R 1: f_{0}$ tuning, this low-amplitude waveform was still observed up to the top of their vocal range despite the use of another resonance tuning $\left(R 2: f_{0}\right)$ in that range (Garnier et al., 2010). These observations indicate that the M3 glottal behavior is not just a weakened version of the laryngeal mechanism M2, due to an absence of resonance tuning. This implies the existence of a laryngeal transition in the high range that is independent from resonance transitions.

With two resonance transitions and the M2-M3 laryngeal transition, there could theoretically be four registers in the high soprano range, above the primo passaggio. This is in accord with R. Miller (2000), who considers a lower middle register over the Eb4-C\#5 range, followed by an upper middle register up to $\mathrm{F \# 5}$ (the average pitch of the secondo passaggio), then an upper register over the $\mathrm{F} \# 5-\mathrm{C} \# 6$ range, followed by a flageolet register above C\#6 (supposed to be equivalent to the whistle register of other authors). The pitches of these register transitions match completely those of the resonance and laryngeal transitions characterized in this study on 12 sopranos. Comparison of our results with Miller's approach to the soprano registers suggests that his lower middle and upper middle registers are very likely to correspond to voice productions with the laryngeal mechanism M2 and that these registers may be distinguished by the absence or presence of $R 1: f_{0}$ tuning below or above C\#5, respectively [see Fig. 9(a)]. Likewise, his upper and flageolet registers probably correspond to voice productions with the laryngeal mechanism $\mathrm{M} 3$, supported by $R 1: f_{0}$ or $R 2: f_{0}$ resonance tuning above C\#6, respectively [see Fig. 9(a)]. The secondo passagio of sopranos would then be associated with the M2-M3 laryngeal transition, and would not necessarily coincide with the lower limit of the $R 1: f_{0}$ resonance tuning (Miller, D.G., 2000; Echternach et al., 2010).

In practice, however, the pitch region of the M2-M3 laryngeal transition was not always found in the middle of the C5-C6 range [i.e., Fig. 9(a) and strategy 2 in Fig. 9(b)], so that there may be two further strategies, in addition to R. Miller's average picture of the soprano registration. Indeed, the M2-M3 laryngeal transition was found as low as C5-D5 in some sopranos, close to the lower limit of the $R 1: f_{0}$ resonance tuning [strategy 1 in Fig. 9(b)]. For others, the M2-M3 laryngeal transition was found as high as C6, close to the upper limit of the $R 1: f_{0}$ resonance tuning [strategy 3 in Fig. 9(b)]. Furthermore, some singers showed the ability, over a subset of the C5-C6 range, to use either the M2 or the M3 laryngeal mechanism, depending on the desired voice quality (full head or fluty resonant).

These observations imply that sopranos may sometimes feel only two register transitions, instead of three, over their high range, above the primo passaggio, and that the register transitions may be of different nature (resonance and/or laryngeal) across singers.
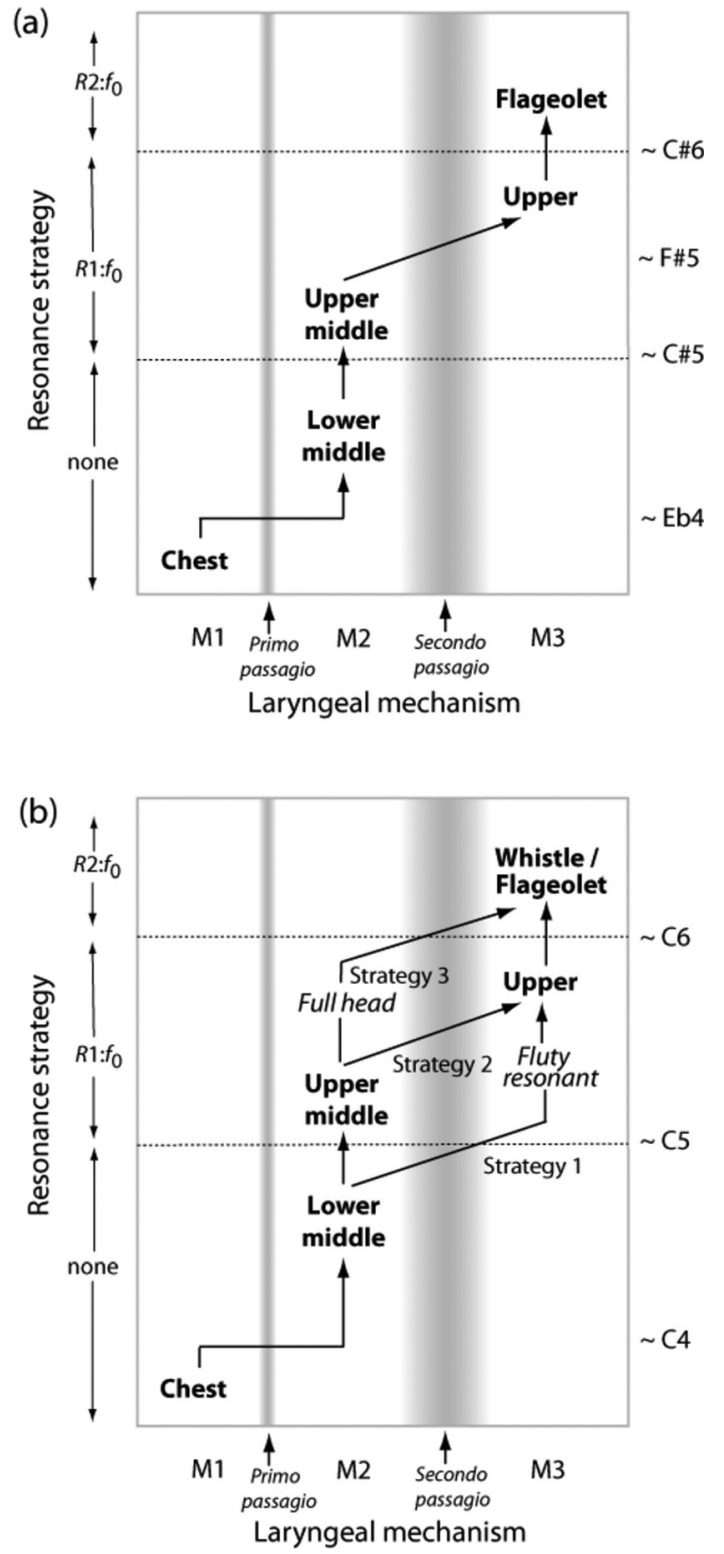

FIG. 9. (a) Idealized schematic indicating the different registers considered by R. Miller (2000) for the soprano voice, and how they may be related to combinations of laryngeal mechanism (M1, M2, M3) and vocal tract adjustment (no tuning, $R 1: f_{0}, R 2: f_{0}$ ). (b) Representation of the three different strategies observed on the 12 sopranos of this study to produce the C5-C6 range. As a result, sopranos may feel different number and nature of register transitions over their high range, above the primo passaggio.

These observations also question the pitch range of the middle upper and the upper registers of R. Miller (2000). Indeed if these registers are associated with the same laryngeal and resonance properties over their whole range, then our results support the idea that the middle upper register could be extended to higher pitches (up to C6) and that the upper register could be extended to lower pitches (down to C5).

\section{CONCLUSION}

Three regions of pitch jumps or instabilities were observed in the high soprano range, above the M1-M2 
transition or primo passaggio. However, EGG and direct endoscopic investigations revealed only one main laryngeal transition in that range, the transition between the M2 and M3 laryngeal mechanisms proposed by Roubeau et al. (2009). The range of this laryngeal transition varied among the 12 singers. Its lower limit was between G4 and D\#5 (A\#4 on average). The upper limit, varying between D5 and D\#6, was usually lower for expert singers. Some singers demonstrated an overlap range (within the C5-C6 upper range) within which they could choose to use either the M2 or the M3 glottal behavior, depending on the desired voice quality. Voice breaks (discontinuities in pitch) coincided with the upper limit of the M2-M3 transition during glissandi or decrescendi, as well as with changes between resonance tuning strategies (no tuning, $R 1: f_{0}$ and $R 2: f_{0}$; see Garnier et al., 2010). However, for most singers, the M2-M3 laryngeal transition did not coincide with one of these changes of resonance tuning.

\section{ACKNOWLEDGMENTS}

We thank our volunteer subjects and the Australian Research Council for support.

Behnke, E. (1880). The Mechanism of the Human Voice, 12 ed. (J. Curwen \& Sons, London), pp. 85.

Berry, D. A., Herzel, H., Titze, I. R., and Story, B. H. (1996). "Bifurcations in excised larynx experiments," J. Voice 10, 129-138.

Chuberre, B. (2000). "Les registres et passages dans la voix chantée," ("Registers and passaggios in singing"), Medical thesis, University of Nantes, France.

Echternach, M., Sundberg, J., Arndt, S., Markl, M., Schumacher, M., and Richter, B. (2010). "Vocal tract in female registers-A dynamic real-time MRI study," J. Voice 24, 133-139.

Garnier, M., Henrich, N., Smith, J., and Wolfe, J. (2010). "Vocal tract adjustments in the high soprano range," J. Acoust. Soc. Am. 127, $3771-3780$.

Henrich, N. (2001). "Etude de la source glottique en voix parlée et chantée," ("The glottal source in speech and singing"), Ph.D. thesis, University of Paris, France.

Henrich, N. (2006). "Mirroring the voice from Garcia to the present day: Some insights into singing voice registers," Log. Phon. Vocol. 31, 3-14.

Henrich, N., d'Alessandro, C., Doval, B., and Castellengo, M. (2004). "On the use of the derivative of electroglottographic signals for characterization of nonpathological phonation," J. Acoust. Soc. Am. 115, 1321-1332.

Henrich, N., d'Alessandro, C., Doval, B., and Castellengo, M. (2005). "Glottal open quotient in singing: Measurements and correlation with laryngeal mechanisms, vocal intensity, and fundamental frequency," J. Acoust. Soc. Am. 117, 1417-1430.

Henrich, N., Smith, J., and Wolfe, J. (2011). "Vocal tract resonances in singing: strategies used by sopranos, altos, tenors, and baritones," J. Acoust. Soc. Am. 129, 1024-1035.

Herzel H., and Reuter, R. (1997). "Whistle register and biphonation in a child's voice," Folia Phoniatr. Logop. 49, 216-224.

Joliveau, E., Smith, J., and Wolfe, J. (2004a). "Tuning of vocal tract resonance by sopranos," Nature 427, 116.

Joliveau, E., Smith, J., and Wolfe, J. (2004b). "Vocal tract resonances in singing: The soprano voice," J. Acoust. Soc. Am. 116, 2434-2439.
Keilmann, A., and Michek, F. (1993). "Physiologie und akustische Analysen der Pfeifstimme der Frau," ("Physiology and acoustical analysis of the female whistle voice"), Fol. Phoniatr. 45, 247-255.

Miller, D. G. (2000). " Registers in singing: empirical and systematic studies in the theory of the singing voice," Ph.D., University of Groningen, The Netherlands.

Miller, D. G., and Schutte, H. K. (1993). "Physical definition of the "flageolet register," J. Voice 7, 206-212.

Miller, R. (2000). Training soprano voices (Oxford University Press, New York), pp. 15-28.

Pressman, J. J., and Kelenen, G. (1955). "Physiology of the larynx," Physiol. Rev. 35, 506-554.

Rothenberg, M. (1988). "Acoustic reinforcement of vocal fold vibratory behavior in singing," in Vocal Physiology: Voice Production, Mechanisms and Functions, edited by O. Fujimura (Raven Press, New York), pp. 379-389.

Roubeau, B., Castellengo, M., Bodin, P., and Ragot, M. (2004). "Phonétogramme par registre laryngé," ("Voice range profile for each laryngeal register," Fol. Phoniatr.t Logo. 56, 321-333.

Roubeau, B., Chevrie-Muller, C., and Arabia-Guidet, C. (1987). "Electroglottographic study of the change of voice registers," Fol. Phoniatr. 39, 280-289.

Roubeau, B., Chevrie-Muller, C., and Arabia, C. (1991). "Control of laryngeal vibration in register change," in Vocal Fold Physiology: Acoustic, Perceptual, and Physiological Aspects of Voice Mechanisms, edited by J. Gauffin and B. Hammarberg (Singular Publishing Group, San Diego, CA), pp. 279-286.

Roubeau, B., Henrich, N., and Castellengo, M. (2009). "Laryngeal vibratory mechanisms: The Notion of vocal register revisited," J. Voice 23, 425-438.

Shipp, T., and Izdebski, K. (1975). "Vocal frequency and vertical larynx positioning in singers and non-singers," J. Acoust. Soc. Am. 58, 1104-1106.

Shipp, T., Lindestad, P.-A., MacCurtain, F., Walker, J. S., and Welch, G. E. (1988). "Whistle register and falsetto voice (Discussion)," J. Voice 2, $164-167$.

Sonninen, A., Hurme, P., and Laukkanen, A-M. (1999). "The external frame function in the control of pitch, register, and singing mode: Radiographic observations of a female singer," J. Voice 13, 319-340.

Sundberg, J. (1975). "Formant technique in a professional female singer," Acustica 32, 89-96.

Sundberg, J. and Skoog, J. (1997). "Dependence of jaw opening on pitch and vowel in singers," J. Voice 11, 301-306.

Svec, J., Sundberg, J., and Hertegård, S. (2008). "Three registers in an untrained female singer analyzed by videokymography, strobolaryngoscopy and sound spectrography," J. Acoust. Soc. Am. 123, 347-353.

Thurman, L., Welch, G., Theimer, A., and Klitzke, C. (2004). "Addressing vocal register discrepancies: an alternative, science-based theory of register phenomena," in Second International Conference on Physiology and Acoustics of Singing (Denver, Colorado, CO), pp. 1-64.

Titze, I. R. (1994). "Vocal registers," in Principles of Voice Production (Prentice Hall, Englewood Cliffs, NJ), pp. 252-278.

Titze, I. R., and Hunter, E. J. (2004). "Normal vibration frequencies of the vocal ligament," J. Acoust. Soc. Am. 115, 2264-2269.

Tsai, C.-G., Shau, Y.-W., Liu, H.-M., and Hsiao, T.-Y. (2008). "Laryngeal mechanisms during human 4-khz vocalization studied with CT, videostroboscopy, and color Doppler imaging," J. Voice 22, 275-282.

Van Deinse, J. B. (1981). "Registers," Fol. Phoniatr. 33, 37-50.

Van den Berg, J. (1963). "Vocal ligaments versus registers," NATS Bull. 20, 16-31.

Walker, J. S. (1988). "An investigation of the whistle register in the female voice," J. Voice 2, 140-150.

Wilson, T. A., Beavers, G. S., DeCoster, M. A., Holger, D. K., and Regenfuss, M. D. (1971). "Experiments on the fluid mechanics of whistling," J. Acoust. Soc. Am. 50, 366-372. 